\title{
Derecho penal de la seguridad Los peligros del derecho penal en la sociedad del riesgo ${ }^{1}$
}

Urs Kindhäuser ${ }^{2}$

\begin{abstract}
Resumen: una sociedad que en camino de su progreso (técnico) se genere a sí misma múltiples peligros debe, en compensación, desarrollar los instrumentos de control social que procuren seguridad. A tal efecto se recurre, también, cada vez más al derecho penal, el cual, sin embargo, no puede ejecutar esa tarea sin acortar o incluso renunciar a elementos esenciales del más básico estado liberal de derecho.

Palabras clave: derecho penal, sociedad del riesgo, estado liberal de derecho, delitos de peligro abstracto, legitimidad del derecho penal, criterios de política criminal, reglas probatorias, protección de bienes jurídicos, fines de la pena, culpabilidad por el hecho individual, legislación simbólica, comprensión liberal del ser humano.
\end{abstract}

\section{Introducción}

En una sociedad cambiante también se transforma el derecho penal. Este tipo de transformaciones no tienen nada de espectacular. Ellas son lentas y se llevan a cabo sin contornos precisos, como por sí mismas. De igual forma, quien hoy por hoy fija su atención en los cambios del derecho penal bien podría aun permanecer tranquilamente despreocupado: el núcleo en su totalidad ofrece una sólida apariencia, solo en las áreas marginales parece haber movimiento. Ahora bien, quien de esta forma se sosiega simplifica las cosas en demasía. El llamado del destacado penalista Hellmuth Mayer ${ }^{3}$, elevado hace décadas, a restringir de una vez por todas la penalización permanente, desmedida y, por eso mismo, desdeñosa del valor humano, en favor de una política criminal sensata, científicamente

\footnotetext{
1 Traducción del alemán por Orlando Humberto De La Vega Martinis.

2 Director del Instituto de Derecho Penal de la Universidad de Bonn, profesor de la materia.

${ }^{3}$ Strafrechtsreform für heute und morgen, 1962, pássim.
} 
respaldada y éticamente reflexiva ha ido apagándose sin consecuencias. El derecho penal se expande sin traba. Él es comprendido -tal como lo sostiene un defensor de la tendencia expansionista ${ }^{4}$ - simple y llanamente como el medio para una reducción óptima de molestias. Al respecto no debería perderse de vista que el derecho penal solo encontrará legitimación por referencia a su área nuclear. ${ }^{5}$ En efecto, todo aquel que emprenda la tarea de justificar el derecho penal, lo justificará pensando en asesinato, violación y robo. A nadie se le ocurriría erigir una institución como el derecho penal, ni tampoco justificar la mera necesidad de su existencia, para asegurar, por ejemplo, que los comerciantes observen el deber de llevar libros contables (§ $283 \mathrm{~b}$ StGB). Ahora bien, si a nadie se le ocurriría fundamentar la existencia del derecho penal a causa de la particular dañosidad social del quebrantamiento del deber de llevar libros contables, entonces una forma de comportamiento tal tampoco es merecedora de pena. De allí que la disposición que ordena llevar libros contables constituya un delito "rémora", es decir, un delito que, a diferencia de los delitos del área nuclear jurídico penal, viaja sin poseer el tiquete del merecimiento de pena. Se trata de un polizón al que se le permite acompañar en el viaje a los viajeros legítimos porque (aparentemente) no los estorba, pero que realmente está autorizado a viajar con ellos solo en la medida en que releva al legislador de la tarea de asegurar la observancia del deber de llevar libros contables de otra manera quizás más complicada.

Este polizón no es el único que hay en derecho penal, y tampoco se trata de dos o tres más. Los viajeros sin tiquete son un número amplio y constantemente se están subiendo nuevos. El derecho penal legitimado por referencia al asesinato, la violación y el robo ha cambiado su apariencia. Y esto significa que los que antes eran viajeros sin tiquete ya no son más viajeros sin tiquete $\mathrm{y}$, antes bien, han pagado el importe de su viaje. Es

\footnotetext{
${ }^{4}$ Kratzsch, Verhaltenssteuerung und Organisation im Strafrecht, pág. 407.

${ }^{5}$ Respecto de este planteamiento véase Kindhäuser, GA 1989, pág. 493.
} 
simplemente que en este precio ya no se trata más de una profunda e inhumana intromisión en las esferas de libertad personal de los cociudadanos, del quebrantamiento de las reglas fundamentales de coexistencia bajo formas jurídicas, como sí es el caso en tratándose de asesinato, violación y robo, sino de una molestia en la seguridad en todos las ámbitos de la vida social y estatal. Quien observe la vehemente discusión respecto de la punibilidad del aborto podría ser de otra opinión. Podría pensar que en derecho penal se intenta contestar con gran esfuerzo y paciencia a la pregunta por el injusto y la culpabilidad punibles. Pero esta discusión no constituye el paradigma de comprensión del derecho penal de hoy ni de la actual política criminal. Ella es una excepción, y justamente también porque en dicha discusión se llevan a cabo cruzadas simbólicas ${ }^{6}$ en terreno jurídico penal.

\section{Seguridad mediante la prohibición de puestas en peligro}

Es perfectamente posible que haya muchas razones que expliquen por qué esta o aquella forma de comportamiento haya sido penalizada en el pasado o en la actualidad lo sea. Pero para un análisis del derecho penal como sismógrafo que mide el estado de una sociedad interesa, por sobre todo, un tipo de delito, el cual en las últimas leyes modificatorias del código penal viene ejerciendo un reinado casi que absoluto: los así denominados delitos de peligro abstracto. ${ }^{7}$ Estos delitos no son creados para castigar la

\footnotetext{
6 Básico al respecto Gusfield, Symbolic Crusade. Status Politics and the American Temperance Movement, passim.

${ }^{7}$ En derecho penal se divide la tipicidad de acuerdo a tres clases de delito: delitos de lesión, delitos de peligro concreto y delitos de peligro abstracto. En los delitos de lesión el injusto consiste en un daño que menoscaba sustancialmente un bien jurídico, por ejemplo el homicidio o las lesiones corporales. En los delitos de peligro concreto el injusto radica en la creación de una situación en la cual -desde el punto de vista del bien jurídico- la entrada o ausencia de daño depende exclusivamente del azar, por ejemplo el abandono de una persona desvalida por decrepitud ( $\$ 221 \mathrm{StGB}$ ). En los delitos de peligro abstracto el injusto está únicamente referido a la peligrosidad en general de la forma de comportamiento, de suerte que el comportamiento concreto puede ser absolutamente inofensivo. Así, por ejemplo, la embriaguez en el tráfico rodado (§ 316 StGB) también es punible, aunque el autor solo haya manejado un trayecto corto en una calle desierta. Las raíces dogmáticas de los delitos de peligro abstracto se remontan al antiguo derecho penal policíal o, más exactamente, a las contravenciones
} 
causación de daños concretos, sino para procurar seguridad. O, dicho de otro modo: el injusto de estos delitos no consiste en ningún daño concreto sino en la maximización de inseguridad. Este injusto de maximización de inseguridad puede tener lugar de distintas maneras, por ejemplo mediante una acción con propósitos malévolos ${ }^{8}$ o mediante el menoscabo objetivo de las condiciones de seguridad ${ }^{9}$ en cualquier ámbito posible de vida. Ahora bien, la garantía de seguridad también puede referirse a la protección lateral brindada por actos administrativos o meras disposiciones contravencionales. Con otras palabras: los delitos de peligro abstracto aplacan en todos los ámbitos neurálgicos del estado y la sociedad la -como pomposamente se denomina- necesidad de acciones en la política de seguridad: drogas, subvenciones, economía, impuestos y cargas sociales, protección del medio ambiente, control de la economía exterior y de las armas de guerra, alimentos, terrorismo, paz social y "clima interior de pacificación”, empleo automático de datos, etc. Donde quiera que uno vaya, allí se escucha, a veces a voz en cuello, a veces en susurro, el anuncio del derecho penal: "tranquilos, ya llegué". Y cada vez que algo sale mal se buscan lagunas en el derecho penal, las cuales -independientemente de que realmente se trate de lagunas- son inmediatamente cerradas de manera particularmente intensa.

Por supuesto, el crítico no tiene derecho a desaprobar precipitada y globalmente este desarrollo pues el esfuerzo por lograr seguridad no es en sí mismo ilegítimo. Se trata, sencillamente, de lo cuestionable que resulta el

que antaño se encontraban junto a los crímenes y delitos del derecho penal criminal. Su marcha triunfal inició, no obstante, recién hace dos siglos. Detallado al respecto véase Kindhäuser, Gefährdung als Straftat, pássim.

8 A título de ejemplo el $\S 267$, párrafo 1, primera alternativa, StGB prohibe la creación de un documento falso con la intención de usarlo después con fines de engaño. En consecuencia, el autor también se hace responsable en caso que, inmediatamente después de su creación, procediera a destruir la falsificación. Constitutivo para el injusto es aquí, preponderantemente, la intención.

9 Ejemplificativo en este contexto es la protección de la administración de justicia frente a declaraciones falsas de testigos y peritos mediante los delitos de perjurio consagrados en los $\S \S 153$ y siguientes StGB. En efecto, el que la declaración falsa influyera, así fuera tan solo en una mínima parte, en la decisión judicial, o no, es un aspecto que no tiene incidencia para la puniblidad de la conducta. 
actual ascenso de la seguridad, la materia genuina del derecho de policía y de contravenciones, a la categoría de idea rectora del derecho penal, la posibilidad de que no encuentre allí merecida atención y adecuada realización, o de que su imposición con ayuda del derecho penal resquebraje el marco de política criminal razonable en un estado liberal de derecho. El contexto en el que debe leerse la actual actividad legislativa en materia penal es el contexto de una política de seguridad como expresión de una sociedad que puede denominarse sociedad del riesgo. El derecho penal de la seguridad es entendido, entonces, como una de las condiciones de estabilidad de la sociedad del riesgo.

\section{El ser humano como factor de inseguridad}

Un riesgo es un peligro endógeno y, al mismo tiempo, controlable. Un peligro se puede controlar, en primer lugar, mediante el expediente de asegurarse contra él. El aseguramiento, además, hace llevadero el peligro, tanto a nivel individual como social. Un peligro también puede ser controlado, en segundo lugar, cuando las condiciones para la posible entrada de un daño son cognoscibles y calculables. En particular los sistemas técnicos se transforman de fuente intolerable de peligro a sector tolerable de riesgo cuando se cierra la posibilidad de daño bajo el presupuesto de que las condiciones de seguridad son observadas. De allí surge nítido dónde radica el problema principal: el factor primario de riesgo es el ser humano mismo. El ser humano es el elemento de inseguridad en la sociedad del riesgo a causa de sus debilidades físicas y síquicas, y de todas aquellas cualidades que impiden que engrane en el sistema social y sus subsistemas como un piñón bien aceitado y sin defectos de desgaste.

El no del todo mecanizado ser humano solo puede ser gobernado y socialmente controlado mediante normas. Y entre más grandes sean los (posibles) daños con capacidad de desencadenar reacciones erróneas $u$ otras 
actitudes desviadas al interior de un sistema de riesgos, de mayor peso tendrán que ser las normas que pretendan integrar al ser humano, como factor de inseguridad, en la disciplina de la seguridad. Que por este camino se llega rápidamente al derecho penal, la institución de control social mas formalizada e intensiva, dificilmente puede sorprender. Y que de esa forma el derecho penal se convierte en el instrumento universal de reglamentación y en el garante de la seguridad, se revela simplemente como la conclusión lógica. Pero que el derecho penal realmente tenga el poder para ejecutar esa tarea solo raras veces entra en consideración política. Es como si no hubiera alternativa alguna. Y si el derecho penal -que por lo menos formalmente se puede describir como la más severa represalia- fracasa, ¿quién podrá, entonces, garantizar seguridad frente a conductas desviadas?.

\section{Los límites del derecho penal legítimo}

Pretender la imposición de una política de seguridad tal con argumentos que señalen los puntos débiles que de facto presenta el control social jurídico penal es, en mi opinión, una tarea inútil. Pues de dicho conflicto fáctico solo con dificultad puede extraerse una postura concluyente. Por el contrario, lo decisivo es la existencia de barreras normativas que irrefutáblemente se oponen a la arbitraria tendencia expansionista del derecho penal de la seguridad. El derecho penal no existe para posibilitar el ejercicio desmedido de violencia según el gusto de cada quien. Antes bien, su empleo se encuentra bajo una necesidad enorme de legitimación. En efecto, la pena consiste en la irrogación de un mal -que dado el caso puede incluso destruir la personalidad- como expresión de desaprobación ético social por un comportamiento defectuoso. Y dado que la pena, tanto por su forma como por su naturaleza, interviene masivamente en las esferas de libertad del autor como persona, su imposición está atada a un amplio número de 
presupuestos jurídico estatales que dificultan que el derecho penal se muestre como un medio efectivo de batalla.

Así, en primer lugar, la punibilidad debe estar legalmente determinada ex ante y no tiene derecho a permanecer abierta a futuro mediante la posibilidad de analogía. ${ }^{10} \mathrm{El}$ derecho penal, en segundo lugar, no autoriza intervenciones para la evitación de peligros amenazantes. Por el contrario, él es retrospectivo y presupone la comisión de un injusto. La suya es una reacción post-et-propter-hoc. En tercer lugar, el derecho penal está ligado al principio de proporcionalidad y su implementación formal encuentra cohesión en el (no en última instancia educativo) proceso penal propio de un estado de derecho mediante una profusión de reglas probatorias y de prohibiciones de empleo de pruebas, así como mediante el principio "in dubio pro reo".

La irrogación de un mal -que merma libertad- mediante pena exige, igualmente, también de la política criminal cierto tipo de restricciones, de las cuales por lo menos dos merecen atención especial. En primer lugar, las normas de comportamiento respaldadas mediante pena tienen que servir a la protección de bienes jurídicos. Para que la política criminal pueda, desde esta perspectiva, orientarse a fines, la lesión o puesta en peligro de bienes jurídicos debe, primero, materializarse en un daño empíricamente comprobable y, segundo, el comportamiento relevante debe causar ese daño -también- de manera empíricamente comprobable. Solo entonces se puede prohibir un comportamiento para evitar un daño. Solo entonces puede el derecho penal funcionar, ciertamente, valiéndose de instrumentos. La segunda columna de la política criminal debe tener apropiadamente en

\footnotetext{
10 Por analogía se entiende la aplicación extensiva de un enunciado jurídico a un estado de cosas no regulado, pero similar. De allí que la analogía sea en sí y para sí una importante figura metodológica de la dogmática jurídica, la cual permite mantener al derecho (también el codificado) abierto a nuevos desarrollos no pronosticados legislativamente. Ahora bien, en derecho penal reina el principio inflexible de "nullum crimen sine lege", el cual le garantiza a todo ciudadano que solo son punibles aquellas acciones que al momento del hecho estuvieren claramente definidas como delito. Famosa es la decisión del Tribunal Imperial que no sancionó la sustracción de energía eléctrica como hurto en razón a que la electricidad no era una cosa en el sentido de la prohibición de hurto del $§ 242$ StGB (RGStE 32, 165).
} 
cuenta la exigencia de que la pena presuponga culpabilidad. Culpabilidad es responsabilidad por el comportamiento defectuoso concreto y presupone, así, la imputabilidad subjetiva de todos los constituyentes del injusto. Y solo una culpabilidad realmente grave puede justificar la pena criminal.

\section{Zonas de tensión del derecho penal de la seguridad}

Este derecho penal del estado liberal de derecho junto a sus -recién delineados- fundamentos político criminales no puede ser tomado libre de fricciones y sin constantes pérdidas sustanciales al servicio de una política universal de seguridad. A continuación se mencionarán algunas de las muchas áreas de tensión.

\section{a) Recorte de las exigencias probatorias}

Primera zona: un derecho penal de la seguridad tratará, antes que nada, de suavizar las escrupulosas exigencias probatorias del derecho penal del estado liberal de derecho. El estrecho apego a la ley, el tenor literal como límite interpretativo y la prohibición de analogía son, todos ellos, obstáculos para un precipitado derecho penal de la seguridad. Quizás por esa razón las últimas leyes en materia penal sean ricas en palabras, incluso locuaces, pero no precisas ni exactas, sino llenas de conceptos indefinidos y cláusulas generales. ${ }^{11}$ Adicionalmente, la prueba de que el autor conocía todos los elementos del injusto de su comportamiento (dolo) o que habría podido y

\footnotetext{
11 Un ejemplo de lo anterior es el reciéntemente creado tipo penal de la estafa informática del § 263a StGB, el cual reza: "Quien, con la intención de conseguir para sí o para un tercero una ventaja patrimonial antijurídica, influya en el resultado de una operación de procesamiento de datos mediante la configuración incorrrecta del programa, mediante el empleo de datos incorrectos o incompletos, mediante el empleo no autorizado de datos o, en general, mediante influencia ilícita en el curso del proceso, menoscabando así el patrimonio de otro, será penado...". La pregunta acerca de cómo se configura "incorrectamente" un programa bien podría ser vista como un acertijo que algún día resolverá la jurisprudencia y la ciencia. Mucho más problemático desde el punto de vista del estado de derecho es la fórmula indefinida del "empleo no autorizado de datos". Y la expresión "en general, mediante influencia ilícita" representa, por supuesto, una crasa infracción del principio -anclado a nivel jurídico constitucional- de determinación. Con ella, el legislador le saca el quite de manera inconstitucional a su responsabilidad y le transfiere a los tribunales, a discreción, la facultad de agrupar aquello que sea tenido por adecuado bajo las disposiciones en cuestión.
} 
debido conocerlos (imprudencia) es, en muchas ocasiones, de difícil consecución. Para eludir esas dificultades la legislación se vale de dos trucos. El primero consiste en extraer de la conexión entre imputación y culpabilidad elementos del injusto del hecho para tratarlos como condiciones objetivas $^{12}$ de punibilidad. El segundo consiste en la creación de delitos en los cuales, simple y llanamente, faltan elementos esenciales del injusto que debe ser evitado. Esto último tiene lugar con frecuencia mediante un fraude de etiquetas. Así, por ejemplo, ni la "estafa” crediticia ( $\$ 265$ b StGB) ni la "estafa" de inversión de capitales presuponen que se induzca en error a la víctima, ni tampoco que ella efectúe un traslado patrimonial que le resulte perjudicial, como sí es el caso en tratándose de una estafa "verdadera" (§ 263 StGB). ${ }^{13} \mathrm{Y}$ ambos trucos pueden también combinarse: por ejemplo, en caso de incapacidad de pago el autor será penado por bancarrota (§ 283 StGB) incluso si la iliquidez no es la consecuencia causal de su turbio comportamiento e incluso si él tampoco la previó o pudo siquiera preverla como la consecuencia concreta de su comportamiento. El reproche de culpabilidad que le queda a semejante remanente de delito radica en una gesitón de negocios contraria a los postulados mercantiles, ni más ni menos.

A la lógica de un derecho penal de la seguridad que quiera corresponderle a la necesidad de seguridad de la sociedad pertenece, además, la penalización sectorial de formas de comportamiento cuya relevancia dañina no está demostrada. De conformidad con lo anterior, una

\footnotetext{
12 Aquí se trata de aquellos presupuestos de la punibilidad a los que no tienen porque estar referidos el dolo o la imprudencia del autor. Por ejemplo, quien afirma un hecho difamante de otro es penado cuando el hecho no puede probarse como verdadero (§ $186 \mathrm{StGB})$. Que el autor erróneamente (y dado el caso, con buenas razones) tuviera el hecho por verdadero no tiene ninguna importancia. Ello constituye una evidente infracción del principio de culpabilidad, según el cual el autor tiene que ser responsable de todas las circunstancias relevantes para la punibilidad.

${ }^{13}$ La estafa tiene como objeto una complicada secuencia causal: con la intención de enriquecerse, el autor engaña a su víctima y la induce en error de modo tal que ella ejecuta un traslado patrimonial que la perjudica, permaneciéndole oculto precisamente el carácter económico-perjudicial de su propia acción a causa del error. Es decir, en la estafa el autor convierte a la víctima en un instrumento que se perjudica a sí mismo -a diferencia, por ejemplo, del hurto, donde el autor ocasiona el daño mediante su propia acción de apropiación-. Por el contrario, la estafa crediticia y de inversión de capitales solo conocen la acción objetiva de engaño. En efecto, el ánimo de enriquecimiento del autor fue eliminado del mismo modo en que también lo fue el perjuicio en el propio patrimonio a causa del error, de forma tal que, finalmente, solo queda un remanente con un cuestionable contenido mínimo de injusto.
} 
conducta deberá ser probadamente segura para que esté permitida y no, por ejemplo, probadamente dañina para que esté prohibida. Esta inversión de la carga de la prueba en sede de injusto objetivo, la cual, desde luego, no debe confundirse con una inversión -en perjuicio del autor- de la carga de la prueba contraria al principio de culpabilidad, se obtiene mediante la unión del derecho penal al derecho administrativo. De esta forma se penalizarán los comportamientos que carezcan de permiso administrativo, es decir, los comportamientos que no superen el control administrativo de seguridad.

\section{b) Renuncia a la protección de bienes jurídicos individuales}

Segunda zona: un derecho penal de la seguridad al que no le importa el castigo de daños concretos sino la evitación de molestias sociales también renunciará a la protección de bienes jurídicos individuales. Así, de una parte, se protegen estados que (aún) no existen en lo absoluto y que, en estricto sentido, no constituyen bien jurídico alguno, como por ejemplo la limpieza del agua ( $\$ 324$ StGB), y ello con total independencia del posible impacto que la contaminación pueda tener en la salud humana o en el abastecimiento de agua. Y, de otra parte, se enuncian bienes cuya lesión mediante la conducta concreta del autor no es empíricamente demostrable en el caso particular. Un típico ejemplo consiste en elevar el valor de la capacidad funcional de ámbitos estatales y sociales al nivel de bienes dignos de protección independiente, como ocurre al calificar las operaciones de pago sin dinero en efectivo como el bien jurídico protegido del abuso de tarjeta de cheque, aun cuando aquí el único daño comprobable es el sobrepaso del límite de crédito frente a la respectiva institución financiera. En estos casos la dañosidad concreta mínima de la conducta permanece oculta en la nebulosa invención del bien jurídico. Quizás por ello la creación de bienes jurídicos universales como los recientemente mencionados se beneficie de la existencia de coyunturas complejas y duraderas. 
c) Renuncia a los fines tradicionales de la pena

Tercera zona: tampoco debe perderse de vista que los fines tradicionales de la pena ${ }^{14}$-retribución, prevención especial e intimidaciónhan pasado a un segundo plano en la discusión actual. La así denominada prevención general positiva, abanderada de la estabilización normativa mediante la práctica de fidelidad al derecho, es la doctrina en curso, y no solo por la -ciertamente alta- plausibilidad de dicha teoría, sino también y justamente porque ella encaja con el derecho penal de la seguridad de la misma forma que el punto sobre la i. Particularmente la prevención de delitos mediante medidas educativas sobre el autor, por el contrario, está como se dice informalmente- "out”. ¿Por qué? En caso de asesinato, violación o robo el autor es la causa del conflicto. Que el autor, a su turno, no esté a merced de otros factores -sociedad, comportamiento de la víctima, predisposición- no tiene la menor importancia en tanto él es, de todos modos, la fuente que posibilita el desencadenamiento del conflicto y, dado el caso, su superación. En el derecho penal de la seguridad, en cambio, las condiciones marco del conflicto ya están presentes en muchos espacios: el vaso ya está lleno, simplemente falta que lo desborde una última y decisiva gota -un comportamiento humano defectuoso-. Desastres medioambientales o choques múltiples en la autopista son conflictos sociales que no se pueden explicar con una sola causa o por referencia al autor, y que tampoco pueden superarse mediante la adscripción de culpabilidad por el hecho individual. Además, en estos casos desaparece la frontera entre autor y víctima.

La acción educativa preventivo especial sobre el autor con miras a la evitación de delitos también es en buena medida irrelevante, siempre que, como frecuentemente es el caso en el derecho penal de la seguridad, el autor

\footnotetext{
14 También en el derecho penal contemporáneo continúa siendo controvertida la determinación del fin de la pena. Frente a la teoría absoluta de la retribución (Kant, Hegel), la cual solo acepta la imposición de la pena para el restablecimiento del derecho y la justicia, se encuentran las teorías -orientadas a la evitación de futuros hechos punibles- relativas de la prevención especial (mejoramiento o inocuización del autor) y de la prevención general negativa (intimidación de la generalidad mediante amenaza e imposición de pena). Acerca de esta discusión véase Kindhäuser, GA 1989, pág. 493-507; Neumann/Schroth, Neuere Theorien von Kriminalität und Strafe, pássim.
} 
sea determinado exclusivamente por su rol social. En estos casos la imprudencia relevante se concreta según el rol. Para una resocialización que trascienda el rol no hay espacio.

\section{d) Borrosa culpabilidad por el hecho individual}

Cuarta zona: en un derecho penal de la seguridad resulta harto difícil establecer una relación recíproca entre culpabilidad y daño. En primer lugar, porque -por ejemplo en áreas técnicas- respuestas mínimamente defectuosas pueden ser ya de graves consecuencias. En segundo lugar porque, además, en los típicos delitos de seguridad falta el nexo de proximidad con la víctima $y$, con ello, la energía criminal que demanda la intromisión en las esferas de libertad de los seres humanos que nos rodean. También los sentimientos de culpa pueden ser fácilmente reprimidos, sobre todo porque las consecuencias del hecho -si es que realmente las hubiere, como por ejemplo en tratándose de derecho penal tributario o medioambiental- no afectan de manera palpable al particular y, por lo tanto, tampoco a uno mismo. La responsabilidad por la acumulación y la sumatoria de daños propia del derecho penal de la seguridad no es fácilmente armonizable con la responsabilidad por el hecho individual. Aquí permance un no despreciable resto de "culpabilidad del sistema".

\section{e) Legislación simbólica}

Quinta zona: el derecho penal de la seguridad induce a una política criminal simbólica. El ser humano como factor de inseguridad solo con reservas puede ser gobernado mediante normas jurídico penales. Por ello, quien mediante la creación de leyes de seguridad jurídico penales pretenda, al mismo tiempo, el establecimiento de seguridad real, solo desplazará el problema de la solución fáctica del conflicto al contrafáctico nivel normativo de la negación del conflicto, haciéndole honor al eslogan según el cual "no puede ser, si es que no tiene derecho a ser". 
Pero la legislación simbólica tiene un muy amplio espectro y puede perfectamente resultar útil en sentido político. Pues el peligro no viene prefijado en la consciencia, sino que recién se constituye mediante suposiciones, pronósticos o escenarios de probabilidad teórica y de allí que pueda agrandarse, achicarse o disolverse, dependiendo de cómo se supone que se controlará o, lo que es lo mismo, calculará. Justo en este sentido es que, por ejemplo, un -de facto raquítico- derecho penal medioambiental, el cual prácticamente solo abarca los casos sin importancia de daños medioambientales sin permiso administrativo, puede políticamente presentarse como un confiable y efectivo aporte a la protección del medio ambiente. Algo similar ocurre con el derecho penal informático, cuyo empleo, en lo esencial, se reduce al (ya punible de otro modo) abuso de cajero automático y a la alteración de máquinas tragamonedas.

Y precisamente la mirada al derecho penal medioambiental todavía resulta aleccionadora desde otro punto de vista. El derecho penal, como medio de control social, es innecesario donde quiera que otros sistemas normativos sean reconocidos como vinculantes, por ejemplo la religión o la moral. Debido a la lenta erosión de la vinculatoriedad general de otros sistemas normativos, el derecho penal se ha convertido en la tabla de salvación y se ha transformado de un sistema de coerción externa en un indicador del contenido de la moral social. Aquello que otros sistemas normativos ya no pueden realizar, por ejemplo desarrollar eficazmente una ética de la responsabilidad orientada a la protección del ambiente natural y de las condiciones de vida de las generaciones futuras, le es delegado al derecho, y aquí una vez más al derecho penal, como si fuera lo más natural. De repente el derecho penal ya no es más el garante del mínimo indispensable ético social, sino un manual de responsabilidad social y solidaridad. 


\section{f) Renuncia a la comprensión liberal del ser humano}

Sexta, y especialmente importante, zona: el retrospectivo derecho penal del estado liberal de derecho parte de una determinada comprensión del ser humano de conformidad con la cual el co-ciudadano es alguien en quien se puede confiar y merece, además, ese anticipo de confianza. Un derecho penal con esa comprensión del ser humano se toma su tiempo, espera y solo entra en acción cuando, por así decirlo, el niño ya ha caído al agua. Esto puede resultar peligroso, incluso osado, pero también es autoconsciente. Ese es el clima social en el que el ciudadano mayor de edad verdaderamente existe, en el que también reina la confianza en los mecanismos sociales de autoregulación. El derecho penal de la seguridad brota, por el contrario, de un potencial de miedo social, es desconfiado y desea ponerle fin a las fechorías de su enemigo incluso antes de que sean cometidas.

\section{Conclusiones}

Antes que nada debe resaltarse que la búsqueda de seguridad es legítima. La seguridad es un derecho humano que justifica en importante medida la existencia del estado y su monopolio de la violencia. Así mismo resulta necesario destacar que sería equivocado minimizar, con mayor razón negar, los peligros de la sociedad del riesgo. El estado debe garantizar seguridad en la medida en que ello sea posible y razonable. Esto está fuera de toda duda. Pero de allí no se sigue, ni mucho menos, que el circunspecto y poco flexible derecho penal del estado liberal, con sus reglas probatorias y su principio de culpabilidad, con su alta pretensión ético jurídica y su solo difícilmente honrable exigencia de legitimación, sea el medio adecuado para la superación de los -por doquier al acecho y permanentemente en aumentoproblemas de seguridad de la sociedad del riesgo. En consecuencia, está fuera de toda duda que las normas que procuran seguridad, y entre ellas las 
que prohiben la destrucción de las condiciones de seguridad, son reglas legítimas de la vida en común. Pero de la legitimidad de esas normas de comportamiento no necesariamente se sigue que también sea legítimo imponer siempre su observancia por medio de la pena criminal. El asunto más sensible del derecho penal no viene dado por las normas de comportamiento, sino por las normas de sanción.

La siguiente reflexión es, desde luego, válida: si el derecho penal es la expresión del estado de una sociedad, entonces justamente también el derecho penal en la sociedad del riesgo estará orientado a la consecución de seguridad. Pero en este contexto los hechos tienen una fuerza normativa desbordada, lo cual, sin embargo, no debe convertirse en un obstáculo de cara a la búsqueda de la solución más adecuada. Reforzar el empleo del derecho civil y administrativo ${ }^{15}$, pero también el desarrollo ulterior de un digno de revalorización- derecho contravencional ${ }^{16}$ con un repertorio de medidas orientadas utilitáriamente a la prevención general y distanciado de un reproche de culpabilidad ético social, serían todas alternativas muy prometedoras. Por supuesto, aquí una vez más debe hacerse valer de forma clara e inequívoca el principio de subsidiariedad: el derecho penal solo puede ser la ultima ratio para la solución de conflictos sociales y no, por ejemplo, prima o, incluso, sola ratio. La exigencia de Kant, solo las reglas básicas de la convivencia en común bajo formas jurídicas y su garantía estatal han de ser protegidas por el derecho penal, no debe caer en el olvido.

\footnotetext{
15 El derecho administrativo, el cual sirve a la defensa y precaución del peligro, es el medio legítimo para la consecución de seguridad. No es casual, entonces, que desde la perspectiva de la ciencia jurídico administrativa se critique como inadecuada la protección del medio ambiente mediante disposiciones jurídico penales independientes. Por su parte, el derecho civil también puede jugar un activo papel en la consecución de seguridad mediante una reglamentación de la compensación de daños que sea independiente de la culpabilidad. Ilustrativo en este sentido es, por ejemplo, la nueva ley de responsabilidad por el producto.

${ }^{16} \mathrm{El}$ derecho contravencional es, al igual que el derecho penal, un derecho sancionador represivo, pero que no asocia la sanción (por lo general una multa) con un reproche ético social, de forma tal que no está atado a una culpabilidad personal. Por ello resulta posible, por ejemplo, imponer multas contra personas jurídicas y asociaciones de personas (por ejemplo sociedades por acciones), expropiar ganancias y sancionar el quebrantamiento de los deberes de vigilancia en una empresa.
} 
Para cerrar, todavía debe operar un retorno de principio en un punto adicional. Y ni el legislador, ni la ciencia, ni la justicia están, por ningún motivo, eximidos de transitar por este cuello de botella del derecho penal legítimo: los nebulosos bienes jurídicos universales deben ser traídos de vuelta al territorio conceptual propio del bien jurídico liberal, donde solo entran en consideración como bienes jurídicos aquellas propiedades de personas, cosas e instituciones que sean condiciones demostrables del libre desarrollo de la personalidad en una sociedad. Estos bienes jurídicos también podrían, entonces, dentro de estrechos límites, merecer y disfrutar de la protección mediata de un derecho penal de la seguridad.

\section{Referências}

GUSFIELD, Joseph R. Symbolic Crusade. Status Politics and the American Temperance, 2. Ed., Urbana y Chicago, University of Illinois Press, 1986.

KINDHÄUSER, Urs. Gefährdung als Straftat, Fráncfort del Meno, Vittorio Klostermann, 1989.

. "Personalität, Schuld und Vergeltung" en: GA 1989.

KRATZSCH, Dietrich. Verhaltenssteuerung und Organisation im Strafrecht, Berlín, Duncker \& Humblot, 1985.

MAYER, Hellmuth. Strafrechtsreform für heute und morgen, Berlín, Duncker \& Humblot, 1962.

NEUMANN, Ulfried; SCHROTH, Ulrich. Neuere Theorien von Kriminalität und Strafe, Darmstadt, Wissenschaftliche Buchgesellschaft, 1980. 